\title{
Les représentations sociales de l'écriture chez les chinois
}

\section{Zheng Lihua}

\section{Q OpenEdition}

1 Journals

Édition électronique

URL : http://journals.openedition.org/communicationorganisation/2518

DOI : 10.4000/communicationorganisation.2518

ISSN : $1775-3546$

Éditeur

Presses universitaires de Bordeaux

Édition imprimée

Date de publication : 1 mai 2001

ISSN : 1168-5549

Référence électronique

Zheng Lihua, «Les représentations sociales de l'écriture chez les chinois », Communication et organisation [En ligne], 19 | 2001, mis en ligne le 27 mars 2012, consulté le 01 mai 2019. URL : http:// journals.openedition.org/communicationorganisation/2518; DOI : 10.4000/ communicationorganisation. 2518

Ce document a été généré automatiquement le 1 mai 2019.

(c) Presses universitaires de Bordeaux 


\title{
Les représentations sociales de l'écriture chez les chinois
}

\author{
Zheng Lihua
}

L'écriture, que ce soit sous forme de lettre, de document, de fax ou de message électronique, possède ses propres caractéristiques par rapport à la parole : destinée à la lecture et ayant un support matériel, elle est visible et vérifiable et laisse trace. Ces traits, inhérents à l'écriture et indépendants de ses utilisateurs, peuvent cependant conduire à des représentations sociales différentes selon les cultures, qui varient dans leurs manières de mettre en valeur ces traits. Dans cet article, nous allons essayer d'examiner les représentations sociales que les Chinois se font de l'écriture à partir d'une enquête que nous avons réalisée au cours de l'année $1999^{1}$ et qui porte sur le management interculturel sino-français. Ces représentations nous semblent liées non seulement aux caractéristiques de forme de l'écriture mais aussi aux contextes de son usage.

\section{L'écriture comme moyen de communication marqué}

2 L'écriture est considérée comme plus sérieuse que la parole. Au cours de l'enquête, nous constatons que les Chinois, comme les Français, utilisent l'écriture pour des choses officielles et importantes, comme nous le dit un interprète travaillant dans une entreprise à capitaux mixtes sino-français: "Dans notre société, nous utilisons des correspondances internes pour la communication. Cela concerne des affaires plus ou moins officielles ou des mesures nouvelles à faire connaitre. S'il s'agit de petites choses, on n'a qu'à téléphoner ou parler en face ci face ». L'écriture est donc liée au sérieux qui est lui-même lié à l'officiel et à l'important. Mais qu'est-ce qui est officiel et important? Si, dans le fonctionnement de toute entreprise, on trouve deux types de communication écrite principaux, à savoir la communication écrite verticale, c'est-à-dire qui marque une relation hiérarchique et qui passe de haut en bas ou de bas en haut, et celle horizontale qui circule entre les unités en parallèle et qui connote plutôt une égalité entre ceux qui écrivent et ceux qui reçoivent, il semble que dans une entreprise chinoise, la communication écrite est davantage axée sur 
le vertical. D'un côté, quand l'échelon supérieur veut émettre des décisions, des règlements, ainsi que d'autres documents directifs, il recourt à l'écrit, car l'écrit est plus sérieux et ce sérieux lui permet de renforcer l'autorité ; d'un autre côté, pour soumettre une demande ou un projet pour ratification, l'échelon inférieur ou un individu fait appel à l'écrit, c'est aussi sérieux mais ce dernier sérieux a plutôt pour fonction de marquer le respect. Dans les deux cas, il s'agit d'une communication dissymétrique. L'acte d'écrire, d'ailleurs comme tout acte social, est soumis à des contraintes qui font que n'importe qui ne peut pas écrire n'importe quoi à n'importe qui. Si cela semble vrai pour toutes les cultures, nous avons affaire en Chine à un principe de fonctionnement ayant pour support idéologique le confucianisme. Cette doctrine repose sur les cinq relations auxquelles se ramènent tous les rapports humains et qui toutes impliquent une différence de statut: souverain/sujet, père/fils, aîné/cadet, mari/femme, ami plus âgé/ami plus jeune. Ce principe d'inégalité crée «des rapports d'autorité à l'intérieur de toutes les relations sociales " (Billeter, in KERBRAT-ORECCHIONI, 1994: 75). Si une personne de statut inférieur écrit à une personne de statut élevé non pas pour lui soumettre une demande mais pour lui parler d'un problème, elle est en train de mettre en question les rapports d'autorité. En effet, en Chine, quand cela se produit, il s'agit la plupart du temps des protestations, des plaintes, des mécontentements, etc., qui marquent plus ou moins une situation de crise ou de rupture, car cela sort de l'orbite prédéterminé. C'est probablement cette raison qui explique le fait qu'on évite de s'adresser par écrit à l'échelon supérieur afin de ne pas déplaire. Ainsi, selon l'observation d'un expert français : "Avec les instituts à Guangzhou ou à Beijing. en général, c'est par le téléphone. Quand on écrit, c'est pour dire que quelque chose ne va pas et que pour avancer, il faut faire autre chose. Mais on ne peut pas critiquer les instituts. On a une grosse difficulté à faire une lettre pour dire qu'un dossier de tel institut est mauvais. On le voit, tout le monde le sait, mais on ne l'écrit pas». Il est à souligner que «ne pas écrire » n'est pas une spécificité uniquement chinoise mais constitue bien une stratégie d'évitement propre à toute société où l'écriture exerce un impact sur les relations interpersonnelles : on évite d'écrire pour se protéger ou protéger l'autre. Tout dépend en fait des calculs des gains et des risques que fait l'acteur au moment de l'action, calculs s'effectuant de manières différentes selon les cultures. Seulement, dans la culture chinoise où prime l'harmonie, la stratégie consistant à ne pas écrire et à recourir à la parole nous semble plus utilisée que dans les cultures occidentales.

3 La communication sur l'axe horizontal, c'est-à-dire entre des interlocuteurs de statut plus ou moins égal, se réalise en Chine plutôt par la voie orale qui marque le rapprochement. L'écrit est le plus souvent le résultat des contraintes extérieures. La distance est une de ces contraintes par excellence. En effet, s'il est normal pour une femme d'écrire à son mari quand celui-ci est à l'étranger, il ne l'est pas du tout quand ils se trouvent sous le même toit, sauf en cas de crise de relation. Ainsi, quand la parole est possible, le recours à l'écriture pour une communication entre les personnes sans relation de hiérarchie devient un moyen de communication marqué, c'est-à-dire inhabituel et porteur d'un sens particulier signifiant soit que l'émetteur veut se distancier du récepteur ou le refuser, soit qu'il veut lui imposer une sorte d'autorité en se basant sur le modèle de l'axe vertical. Dans les deux cas, l'écrit connote la distance et le rejet de l'autre. Cette réflexion nous permet de mieux expliquer les difficultés que les experts français ont rencontrées lorsqu'ils voulaient passer des commentaires écrits aux ingénieurs chinois comme le fait remarquer un expert français : "Je faisais quand même des commentaires par écrit, maisj'ai vu 
que c'était pas toujours assez bien senti. Il m'est arrivé de faire un commentaire écrit sur le projet d'un ingénieur. Il m'a répondu que c'est assez sec de faire un commentaire par écrit. Cette fois-ci, j'ai fait mes commentaires par oral et cela s'est très bien passé." Dans ce cas précis, les commentaires écrits marquent non seulement la distance, mais aussi une relation de supériorité de l'auteur car ils font penser aux corrections que le professeur écrit à l'étudiant, les deux inscrits dans un rapport de force incontestablement inégal pour les Chinois. Cet exemple nous permet également d'émettre l'hypothèse selon laquelle si en Chine comme en France on n'écrit moins entre les pairs que dans une relation hiérarchique, l'écriture entre les égaux étant plus ou moins mal sentie, il semble que dans la culture chinoise où la relation entre les pairs, régie par le principe d'inégalité mentionné plus haut, n'est pas réellement développée mais se trouve implicitement hiérarchisée ${ }^{2}$, l'écrit au sein de cette relation acquiert une connotation de distance plus forte que dans la culture française.

\title{
L'écriture liée à l'autre
}

On dit en français «Les paroles s'envolent et les écrits restent.» Un proverbe chinois dit également: « kou shuo wu ping, luo bi wei zheng» (les paroles ne laissent pas de trace et les écrits donnent preuve). Dans les deux cas. on insiste sur la fonction de preuve de l'écriture. Cette fonction est basée sur une des principales caractéristiques de l'écriture: la trace. Tout ce qui est tracé reste. Cela paraît être une vérité dans toutes les sociétés. Mais si les écrits restent, est-ce que les paroles s'envolent toujours? Et est-ce que ces écrits peuvent toujours servir de preuves ? Pour répondre à ces questions, il nous faudrait nous interroger sur les contextes d'utilisation de ces deux moyens de communication qui nous semblent différents selon les cultures et sur la répartition de fonctions entre la parole et l'écriture qui semblent également varier d'une culture à l'autre.

Les deux exemples suivants, tirés de notre enquête et relevés tous deux du contexte chinois, montrent une contradiction dans l'usage de l'écriture :

\begin{abstract}
"L'année dernière, j'ai fait des travaux pour mon nouvel appartement. Une amie m'a prêté 50000 yuans. Elle me les a apportés dans un sac plastique. Je l'ai remerciée, pris le paquet et l'ai mis dans ma chambre, sans les avoir comptés. Je n 'ai pas donné de reçu écrit et mon amie ne me l'a pas demandé. En comptant les billets après le départ de l'amie, j'ai découvert un billet de plus. Je ne pouvais pas lui dire que j'avais trouvé un billet de plus, car cela trahirait le fait que j'ai compté après son départ. J'ai dû lui dire qu'à la banque, l'employée avait trouvé un billet de plus. Il y a deux mois, je lui ai remboursé l'argent. " (une sous-chef dans une entreprise).

"Tout ce qui écrit, par exemple, des contrats, des reçus, des récépissés, est très important, car les commerçants chinois $n$ 'ont pas suffisamment de crédit. Par exemple, une fois, un de nos livreurs a oublié de faire signer le bon de livraison au Magasin Xindaxin qui a refusé de payer les marchandises livrées» (directrice adjointe d'une société chinoise).
\end{abstract}

Ces deux exemples représentent deux cas extrêmes. Dans le premier exemple, tout est fondé sur la parole et cette parole ne s'envole pas, car l'interviewée a bien remboursé l'argent emprunté. En fait, dans ce premier cas, la puissance de garantie ne se trouve pas dans la parole mais dans la relation derrière la parole, c'est-à-dire la relation d'amitié et de confiance. C'est cette relation qui sert de fondement à l'acte de prêter et à l'acte de rembourser qui auront pour effet, à leur tour, de renforcer cette relation. Dans cette situation, l'absence de l'écriture constitue la force du jeu : l'une a prêté sans papier écrit, c'est-à-dire au risque de non remboursement et l'autre a payé sans preuve écrite, soit 
avec la possibilité de renier. La relation se voit ainsi renforcée avec cette épreuve représentée par l'absence de l'écriture. Si l'écriture intervenait, elle risquerait de tout détruire. Imaginons qu'il y ait une reconnaissance de dette et que le remboursement se fassent également sans problème. Les comportements seraient interprétés tout autrement : si l'une a prêté, c'est en partie parce qu'il y a la garantie de remboursement ; si l'autre a remboursé, c'est partiellement parce que qu'elle ne pouvait pas renier. La relation de confiance serait ainsi atténuée. On voit tout de suite que ce fonctionnement volontairement sans écriture ne marchera pas dans le deuxième exemple où l'écrit sert de garantie dans les relations commerciales. Comment expliquer cette absence de l'écriture souhaitée dans le premier cas et cette exigence de l'écriture poussée à l'extrême dans le deuxième cas? Entre ici en jeu un autre principe de la culture chinoise, celui de distinction entre fin-groupe et l'out-groupe.

7 Si dans toute société, l'individu est inévitablement un membre d'une certaine communauté, le sens communautaire acquiert une importance particulièrement grande en Chine. Dans la culture chinoise, chaque individu trouve sa place dans le réseau qu'il forme avec un autre ou les autres. Cette place comporte un nom : il est fils par rapport à ses parents, mari par rapport à sa femme, père par rapport à ses enfants, et ainsi de suite. Chaque nom a ses devoirs. Les noms s'accumulent et les devoirs s'additionnent. L'individu dépend d'autrui pour réaliser sa valeur sociale car cette valeur est définie par le groupe et non pas par lui-même. Son image est contrôlée par l'opinion du groupe. Le confucianisme définit ainsi l'homme social par le mot ren dont la composition est ici révélateur : homme + deux, c'est-à-dire qu'on ne peut définir un homme que par rapport à un autre homme. D'ailleurs, ce sont les autres (le groupe, la société, etc.) qui décident qu'un homme est «bon» ou «mauvais». En effet, en chinois, l'expression ren pin signifie "qualité, personnalité de l'homme.» Le graphisme pin est composé de trois bouches. La qualité d'un homme est donc déterminée par les paroles des autres (ZHENG. 1995 : 60). Ainsi, la régularisation entre les individus est basée sur le contrôle du groupe et les comportements d'un individu sont sanctionnés par le regard ou le dire des autres. Mais les termes le groupe et les autres restent à préciser. Il s'agit du groupe auquel l'individu appartient, c'est-à-dire l'in-groupe pour lui et des autres qui forment avec lui un réseau, c'est-à-dire qui ont le pouvoir de définir son image. Il est pourtant à noter que la distinction entre l'in-groupe et l'out-groupe n'est pas établie une fois pour toutes et qu'une personne de l'out-groupe pourra devenir un membre de l'in-groupe, si elle entre dans le réseau. Si cette distinction entre l'in-groupe et l'out-groupe existe dans toutes les sociétés, il semble que chaque société a sa façon de gérer la relation au sein de l'in-groupe et celle avec l'out-groupe et qu'en Chine, cette distinction joue un rôle particulièrement important, car elle fonctionne comme un principe organisateur des comportements des acteurs sociaux. En effet, une des caractéristiques des Chinois est le particularisme, c'està-dire qu'ils se comportent souvent différemment selon qu'ils se trouvent en présence de l'in-groupe ou de l'out-groupe. Bien qu'on puisse également observer cette différence comportementale chez les Français en fonction de la présence du groupe, elle semble moins poussée, atténuée par le principe de généralisme valorisé dans la culture française.

Une particularité de la relation de l'in-groupe, c'est qu'elle est fondée sur la solidarité et la confiance et qu'elle les présuppose. On n'a pas besoin de preuve écrite car les liens sont supposés suffisamment forts pour fonctionner sans contrôle extérieur. De ce fait, le recours à l'écrit à l'intérieur du groupe peut connoter la méfiance et le rejet. Ainsi, un Français qui voulait épouser une jeune fille chinoise et lui faire signer un contrat de 
mariage écrit a été étonné de recevoir une réaction émotionnelle de celle-ci : « Mais tu ne m'aimes pas!", alors que pour lui, il s'agissait d'une procédure normale. En effet, « en France et dans d'autres pays qui vivent sous le Code Napoléon, les contrats écrits concernant la propriété et la répartition de celle-ci sont une condition sine qua non" (GOODY, 1986 : 148). Mais pour les Chinois, les contrats de mariage écrits partent de la méfiance. Ils cherchent à définir les frontières entre les époux et les séparent par conséquent, ce qui est à l'encontre du principe chinois voulant que le mari et la femme s'unissent et se fusionnent en une seule personne. Ils font penser également au divorce, ce qui constitue déjà un très mauvais augure de l'union conjugale. De même, un directeur chinois de l'entreprise était furieux contre un subordonné chinois qui est venu lui demander un écrit pour une chose qu'il lui avait promise oralement, car demander un écrit, c'est mettre en doute le crédit de l'autre et porter atteinte à sa personnalité. Les preuves écrites ne sont nécessaires qu'avec les personnes de l'out-groupe qui échappent au contrôle de notre groupe. L'écriture est liée à l'autre.

Une autre particularité de la relation de l'in-groupe, c'est que son mode de régularisation est fondé sur l'implicite, ce qui constitue une autre raison de rejet de l'écriture, car justement. l'écriture laisse trace et rend tout explicite. La confiance au sein du couple, l'affection entre les membres de famille, la solidarité entre les personnes appartenant au même groupe sont considérées comme acquises par définition et n'ont même pas besoin d'être verbalisées. Un exemple : une étudiante chinoise aux États-Unis a écrit une fois à ses parents en Chine en terminant sa lettre, avec, à la manière américaine, l'expression «Je vous aime » en gros caractères. Ses parents ont été alertés et ont téléphoné tout de suite à leur fille pour s'informer de ce qui s'était passé, car cette affection envers les parents a besoin d'être explicitée seulement en cas de crise extrême où on sait qu'on va se séparer éternellement de ses parents (source : journaux).

On voit par là que dans la société chinoise, la gestion des relations interpersonnelles à l'intérieur du groupe est basée sur la non-trace et l'implicite et que l'écriture ne convient pas parce que, justement, elle laisse trace et est trop explicite. Ce système de contrôle du comportement des membres du groupe semble différent de celui en Occident qui utilise l'écriture et la signature. Ainsi que le fait remarquer R. Harris : « la signature fournit un puissant mécanisme d'intégration, au moyen duquel toute société qui possède une tradition écrite peut contrôler le comportement de ses membres " (HARRIS, 1993 : 201). Quelle est. en Chine, le système de régularisation des relations avec l'out-groupe ? Comme nous n'avons pas d'emprise sur les personnes appartenant à l'out-groupe, qui peuvent ne pas se soucier des commentaires de notre groupe, la seule garantie, c'est l'écriture qui laisse trace. Mais est-ce que toute trace est valable? En fait, dans toute écriture, que ce soit en Chine ou en Occident, la trace toute seule n'est pas suffisante pour servir de preuve, car il faut quelque chose qui identifie l'origine de l'écriture et qui la valide dans sa fonction de preuve. C'est là où les différences émergent. Si beaucoup de cultures utilisent la signature personnelle et le tampon d'une collectivité pour valider un document écrit, il semble que toutes deux ne représentent pas partout les mêmes valeurs. En Chine, le tampon, qui a derrière lui la caution d'une collectivité, pèse beaucoup plus lourd que la signature, alors qu'en Occident, la signature qui implique une responsabilité personnelle du signataire vis-à-vis de sa signature semble l'emporter sur le tampon où l'on ne voit pas clairement la responsabilité. Si en Chine, un seul tampon rouge peut suffire et qu'en Occident, une seule signature peut suffire, l'inverse ne semble pas vrai lorsqu'on change de pays. Il est pourtant à souligner que l'usage de la signature ou du tampon dépend 
largement de la situation concrète, de la nature du document, et du degré de confiance que les acteurs ont les uns à l'égard des autres. En Chine, quand on laisse un message, c'est plutôt la signature qui est règle; de même, en France, lorsqu'il s'agit d'un document officiel comme un diplôme ou un contrat d'une société, le tampon est plus ou moins obligatoire. La différence culturelle nous semble résider moins dans l'opposition entre la signature et le tampon que dans la structuration des contextes, dans les représentations de ce qui est officiel et des degrés d'exigence demandant une signature, ou un tampon ou les deux en même temps et dans les attitudes que les uns ont vis-à-vis des autres. Ainsi, selon une directrice adjointe chinoise : "Nous acceptons la signature de nos clients européens pour envoyer les marchandises et faire payer après. Mais si c'est une société chinoise, nous ne pouvons pas accepter la signature personnelle. Il faut le tampon de la société, même pour les sociétés avec qui on a de très bonnes relations de coopération et qui ont un bon crédit. Avec le tampon, on ne peut pas renier. Mais si l'on a seulement la signature de l'acheteur sans le tampon de la société, la société ne reconnaît pas si l'acheteur n'est pas là. »Cet exemple nous révèle les attitudes de cette société chinoise à l'égard des clients occidentaux et des clients chinois appartenant en fait tous à l'out-groupe. attitudes devant provenir des expériences accumulées dans le commerce mais qui nous renvoient les images que les Chinois se font de la nature de l'out-groupe occidental et de celle de l'out-groupe chinois qui semble beaucoup plus difficilement contrôlable que le premier. Les propos de cette directrice adjointe reflètent aussi en partie les représentations que les commerçants chinois se font de la signature et du tampon, chinois ou occidentaux. Nous voyons également par là qu'au sein de la culture chinoise, dans l'interaction avec un individu appartenant à l'out-groupe et censé échapper à notre emprise, nous cherchons encore recours auprès de la collectivité qui exerce un contrôle sur lui. Un coup d'œil sur la forme de la signature nous confirme cette idée sur le recours chinois à la force du groupe dans la validation des documents écrits. Du point de vue sémiologique, une signature possède essentiellement deux caractères : le caractère autocentrique et celui de marque d'identification (HARRIS : 1993 : 205). Une signature occidentale nous semble être d'abord la marque d'un individu indépendant des autres, car on insiste sur son caractère autocentrique qui pousse le scripteur à chercher une configuration unique et personnelle et à inventer effectivement son propre signe écrit en toute liberté, ce qui fait que souvent, les signatures occidentales sont illisibles pour les autres. Et pour compenser cette illisibilité, le signataire met audessus ou au-dessous de sa signature, son nom imprimé en toutes lettres ou écrit en majuscules. Une signature chinoise est avant tout la marque d'une personne faisant partie du groupe et on insiste sur son caractère de marque d'identification. Elle doit être reconnaissable et lisible par les autres. La règle de lisibilité prime celle d'originalité.

11 Revenons à la question posée au début de l'article concernant les valeurs de l'écrit et de la parole. Nous voyons à travers les analyses ci-dessus que dans la culture chinoise, l'interaction parmi les personnes faisant partie du même groupe est fondée sur la confiance. Dans ce cas-là, les paroles, au lieu de s'envoler, constituent les fondements des jeux sociaux. Quand il s'agit de l'out-groupe, non seulement les paroles perdent leurs valeurs, mais aussi les écrits peuvent ne pas fonctionner comme preuves s'ils ne sont pas validés et appuyés par une collectivité. En d'autres termes, en situation in-groupe, la parole peut suffire bien qu'elle ne laisse pas de trace alors que dans les relations avec l'out-groupe. même l'écriture peut ne pas suffire bien qu'elle reste.

12 Les réflexions ci-dessus nous conduisent à deux conclusions. Premièrement, la parole et l'écriture constituent, l'une comme l'autre, des réponses aux mêmes problèmes de 
communication posés à toutes les cultures. Ce qu'il faut voir, comme le souligne L.J Calvet, «c'est que la parole régit tout d'une certaine façon, qu'elle a apporté une réponse aux problèmes de la société, et que l'écriture a apporté une autre réponse » (1984:120). Ce qui fait la différence entre les cultures, c'est que «chaque problème de communication, dans une société humaine, trouve sa réponse sous une forme ou sous une autre » (ibid.: 8). Deuxièmement, les différences entre deux sociétés résident moins dans l'opposition entre la parole et l'écriture qui ne représentent que des moyens de résolution de problèmes que dans les valeurs culturelles qui les sous-tendent, comme ici l'individualisme d'un côté qui prime dans les sociétés occidentales et le collectivisme de l'autre côté qui domine dans la société chinoise, dans la structuration des contextes concrets et dans les représentations sociales que chaque culture se l'ait de la parole et de l'écriture.

\section{BIBLIOGRAPHIE}

CALVET, L.J., La tradition orale, Paris : Presses Universitaires de France, 1961.

GOODY, J., La logique de l'écriture. Aux origines des sociétés humaines, Paris : Armand Colin, 1986.

HARRIS, R., La sémiologie de l'écriture, Paris : CNRS Editions, 1993.

KERBRAT-ORECCHIONI, C, Les interactions verbales, Tome III, Paris : Armand Colin. 1994.

ZHENG, L.H., Les Chinois de Paris et leurs jeux de face, Paris : L'Harmattan, 1995.

\section{NOTES}

1. Cette enquête a été réalisée par le Centre des Recherches sur l'Interculturel de l'Université des Etudes étrangères du Guangdong dirigé par $M$. Zheng Lihua. Ont participé à cette enquête $\mathrm{M}$. Cao Yongqiang, Mlle Xiang Jing. Mlle Chen Ying, Mlle Ouyang Junyi et Mlle Séverine Enjolras.

2. Dans la société chinoise, même entre les amis, il existe une inégalité de statut implicite. Autrefois, les meilleurs amis avaient l'habitude de s'adapter en frères pour se nouer dans une relation entre l'aîné et le cadet, relation intrinsèquement inégale en Chine.

\section{RÉSUMÉS}

L'écriture, que ce soit sous l'orme de lettre, de document, de fax ou de-message électronique, possède ses propres caractéristiques par rapport à la parole : destinée à la lecture et ayant un support matériel, elle est visible et vérifiable et laisse trace. Ces traits, inhérents à l'écriture et 
indépendants de ses utilisateurs, peuvent cependant conduire à des représentations sociales différentes selon les cultures, qui varient dans leurs manières de mettre en valeur ces traits. La parole et l'écriture constituent, l'une comme l'autre, des réponses aux mêmes problèmes de communication posés à toutes les cultures. Ce qui fait la différence entre les cultures, c'est que chaque problème de communication, dans une société humaine, trouve sa réponse sous une forme ou sous une autre.

Written materials, either a letter or a document or a fax, do have some characteristics in common. For example, they are written for reading, have a material carrier, can be perceived and traced. These are the innate characteristics of written language, which do not have a close relation with the language user. But the concept of written language that is aroused by the above characteristics varies from culture to culture. Both spoken and written language are necessary communicative means for any society that has a writing system; they can solve different communicative problems sprouting in society. The difference among cultures lies in their different preferences when choice is made between spoken and written language in solving the same problem.

\section{INDEX}

Mots-clés : écriture, parole, représentation

\section{AUTEUR}

\section{ZHENG LIHUA}

Lihua ZHENG, Docteur en sciences du langage de Paris V-Sorbonne, professeur de sociolinguistique à l'Université des Études étrangères du Guangdong (Chine), directeur du Centre de recherches sur l'interculturel, travaille sur la communication dans ses perspectives interpersonnelles et interculturelles. Il est l'auteur de Les Chinois de Paris et leurs jeux de face (L'Harmattan, 1995), de Langage et interactions sociales (L'Harmattan. 1998) et de Chine-France, approches interculturelles en économie, littérature, pédagogie, philosophie et sciences sociales (L'Harmattan. 2000). 